\title{
Unique patterns of tumor growth related with the risk of lymph node metastasis in papillary thyroid carcinoma
}

\author{
Chan-Kwon Jung ${ }^{1}$, Yoon-Goo Kang ${ }^{1}$, Ja-Seong Bae ${ }^{2}$, Dong-Jun Lim ${ }^{3}$, Yeong-Jin Choi ${ }^{1}$ and \\ Kyo-Young Lee ${ }^{1}$ \\ ${ }^{1}$ Department of Hospital Pathology, The Catholic University of Korea, Seoul, Republic of Korea; ${ }^{2}$ Department \\ of Surgery, The Catholic University of Korea, Seoul, Republic of Korea and ${ }^{3}$ Department of Internal Medicine, \\ College of Medicine, The Catholic University of Korea, Seoul, Republic of Korea
}

\begin{abstract}
Although many attempts have been made to predict the occurrence of lymph node metastases from papillary thyroid carcinoma, there are currently no reliable means to accurately predict cervical nodal metastasis. In this study, we present a novel prediction system for the lymph node metastasis based on the histological and cyclin D1 staining features. The frequency of lymph node metastases from a series of 210 papillary thyroid carcinomas was analyzed according to the clinicopathological variables, cyclin D1 staining patterns and BRAF $F^{\mathrm{v} 600 \mathrm{E}}$ mutation in tumor tissue. A total of $113(54 \%)$ patients had lymph node metastasis. Cyclin D1 was constantly expressed at the invasive tumor front and revealed well-defined isolated glands of tumor cells in the extratumoral region (isolated glands) and laterally spreading tubular growth along the fibrous septa around the invasive front of the tumor (lateral tubular growth). Upon univariate analysis, an age of less than 45 years $(P<0.001)$, tumor size of $10 \mathrm{~mm}$ or more $(P<0.001)$, non-follicular variant $(P=0.005)$, invasive growth pattern $(P=0.007)$, extrathyroid extension $(P=0.006)$, isolated glands $(P<0.001)$, lateral tubular growth $(P<0.001)$ and tumor multiplicity $(P=0.005)$ predicted lymph node metastasis, whereas $B R A F^{\mathrm{V} 600 \mathrm{E}}$ mutation did not. Upon multivariate analysis, age $(P=0.001$, odds ratio $(O R)=5.146)$, tumor size $(P=0.034$, $O R=3.119)$, isolated glands $(P<0.001, \mathrm{OR}=21.042)$ and lateral tubular growth $(P<0.001, \mathrm{OR}=24.652)$ were found to be strong independent predictors of lymph node metastasis. Cyclin D1 staining of papillary thyroid carcinoma is very useful for identifying the intrathyroidal spreading or multifocality of the tumors. Tumor growth patterns verified by cyclin D1 staining can be used for the identification of papillary thyroid carcinomas with metastatic potential. Modern Pathology (2010) 23, 1201-1208; doi:10.1038/modpathol.2010.116; published online 11 June 2010
\end{abstract}

Keywords: carcinoma; cyclin D1; lymph nodes; neoplasm metastasis; papillary; thyroid neoplasms

The incidence of thyroid cancer has been rapidly increasing in Korea. Specifically, the age-standardized incidence rate of thyroid cancer increased from 7.2 per 100000 in 1999 to 23.8 per 100000 in 2005 and the annual percentage change was $24.8 \% .^{1}$ Papillary thyroid carcinoma is the most common type of thyroid malignancy and accounts for about $65-88 \%$ of all thyroid cancers in the United States. ${ }^{2}$

Correspondence: Dr Y-J Choi, MD, PhD or Dr C-K Jung, MD, PhD, Department of Hospital Pathology, Seoul St Mary's Hospital, College of Medicine, The Catholic University of Korea, 505 Banpo-dong, Seocho-gu, Seoul 137-701, Korea.

E-mail: ckjung@catholic.ac.kr or mdyjchoi@catholic.ac.kr

Received 26 February 2010; revised 30 April 2010; accepted 6

May 2010; published online 11 June 2010
However, in Korea, papillary thyroid carcinoma accounts for up to $95 \%$ of thyroid cancers. ${ }^{3}$

Papillary thyroid carcinoma tends to metastasize to the cervical lymph nodes during the early stages of the disease. ${ }^{4,5}$ Cervical lymph node metastases are found in $30-90 \%$ of patients at the time of diagnosis. ${ }^{5}$ Nodal metastases increase the risk of loco-regional recurrence and cancer-specific mortality in older patients ( $>45$ years) and distant metastases. ${ }^{5,6}$ Therefore, reliable prognostic factors are needed to assess the risks of lymph node metastases in papillary thyroid carcinoma patients and adapt the initial therapy and follow-up schemes.

Cyclin D1 is frequently overexpressed in thyroid cancer, but not expressed in normal thyroid 
follicular cells. ${ }^{7,8}$ Overexpression of cyclin D1 has been identified as an independent risk of lymph node metastasis in papillary thyroid carcinoma. ${ }^{7,8}$ However, Ito et $a l^{9}$ could not identify any association between high cyclin D1 expression and lymph node metastasis in papillary thyroid microcarcinoma. In some studies, cyclin D1 expression was not an independent predictive factor for lymph node metastasis during multivariate analysis, although it has been found to be associated with lymph node metastasis in univariate analysis. ${ }^{10,11}$

$B R A F^{\mathrm{V} 600 \mathrm{E}}$ mutation has frequently been detected in papillary thyroid carcinoma, but has not been identified in benign thyroid lesions. ${ }^{12,13}$ In Korea, the prevalence of the $B R A F^{\mathrm{V} 600 \mathrm{E}}$ mutation accounts for up to $87 \%$ of all cases of papillary thyroid carcinoma. ${ }^{3,14}$ Xing et $a l^{13}$ included 28 studies in a comprehensive meta-analysis and identified a significant association of $B R A F^{\mathrm{V} 600 \mathrm{E}}$ mutation with lymph node metastasis (odds ratio $(\mathrm{OR})=1.83 ; 95 \%$ confidence interval $=1.58-2.13$ ). However, another meta-analysis showed no association between lymph node metastasis and $B R A F^{\mathrm{V} 600 \mathrm{E}}$ mutation $(\mathrm{OR}=1.500 ; 95 \%$ confidence interval $=0.99-2.27)$. In Korean patients, $B R A F^{\mathrm{V} 600 \mathrm{E}}$ mutation was not associated with lymph node metastasis. ${ }^{11,15,16}$ Thus, additional studies are necessary to investigate the clinicopathological significance of the $B R A F^{\mathrm{V} 600 \mathrm{E}}$ mutation in a larger series of papillary thyroid carcinomas.

This study was conducted to investigate the predictive factors for lymph node metastasis in patients with papillary thyroid carcinoma by analyzing clinicopathological parameters, cyclin D1 staining patterns and $B R A F^{\sqrt{ } 600 \mathrm{E}}$ mutation status.

\section{Materials and methods}

This study was approved by the Institutional Review Board of Seoul, St Mary's Hospital, the Catholic University of Korea (approval number: KC09EISI0355).

\section{Patients}

A total of 210 patients (174 females and 36 males) who underwent total thyroidectomy and neck lymph node dissection for papillary thyroid carcinoma at Seoul St Mary's Hospital between October 2008 and June 2009 were retrospectively analyzed. All patients were diagnosed with papillary thyroid carcinoma preoperatively by fine needle aspiration cytology or intraoperatively on the basis of frozen tissue sections. There was no history of radiation exposure in any patients. The age of the patients at the time of surgery ranged from 22 to 75 years (mean 48 years). In cases with multiple tumor foci, the largest tumors were regarded as the primary lesions. All specimens were collected from patients after informed consent had been obtained in accordance with the institutional guidelines of our hospital.

\section{Immunohistochemistry}

Immunostaining was conducted on $4-\mu$ m-thick sections of formalin-fixed, paraffin-embedded tissue blocks at the plane of the largest dimension of the tumor. Slides were placed in a $60^{\circ} \mathrm{C}$ oven for $1 \mathrm{~h}$ and then deparaffinized in xylene and rehydrated through graded ethanol to water. Antigen retrieval was performed using $0.01 \mathrm{M}$ citrate buffer ( $\mathrm{pH}$ 6.0) by heating the sample in a microwave vacuum histoprocessor (RHS-1, Milestone, Bergamo, Italy) at a controlled final temperature of $121^{\circ} \mathrm{C}$ for $15 \mathrm{~min}$. The endogenous peroxidase activity was then blocked by incubating the slides in 3\% hydrogen peroxide in methanol for $10 \mathrm{~min}$. Slides were then placed in an automated immunohistochemical stainer (Lab Vision Autostainer LV-1; LabVision/ Neomarkers, Fremont, CA, USA) for use with immunohistochemistry and incubated for $30 \mathrm{~min}$ with cyclin D1 rabbit monoclonal antibody (Clone SP4, 1:100, Thermo Scientific, Fremont, CA, USA). The primary antibody was diluted in DAKO Antibody Diluent (DAKO, Carpinteria, CA, USA) with background-reducing components. Next, they were detected using the Envision Plus System (DAKO). The immunoreaction was developed with diaminobenzidine (DAKO) for $5 \mathrm{~min}$, after which it was counterstained with hematoxylin. Sections of a mantle cell lymphoma were used as a positive control in all staining runs. As a negative control, slides were stained without the primary antibody. Nuclear cyclin D1 staining was considered to be a positive result. Because all tumors that expressed cyclin D1 showed moderate-to-strong nuclear staining intensity, we only graded the distribution of positive tumor cells in tumors as follows: grade 1, $1-10 \%$; grade $2,11-25 \%$; grade $3,26-50 \%$; grade 4 , $51-75 \%$; grade $5,76-100 \%$.

\section{Genomic DNA Extraction from Tumor Tissue}

Genomic DNA was extracted from two $10-\mu \mathrm{m}$-thick paraffin sections containing a representative portion of each tumor block using the QIAamp DNA Mini kit (Qiagen, Hilden, Germany). Neoplastic areas were manually microdissected from the tissue sections.

\section{Sequencing of Exon 15 of the BRAF Gene}

A 224-bp fragment of exon 15 of the BRAF gene was amplified by polymerase chain reaction (PCR) using the forward primer $\left(5^{\prime}\right.$-TCATAATGCTTGCTCTGA TAGG- $3^{\prime}$ ) and reverse primer (5'-GGCCAAAAATT TAATCAGTGGA-3'). The PCR conditions were as follows: 35 cycles of $30 \mathrm{~s}$ at $94^{\circ} \mathrm{C}, 30 \mathrm{~s}$ at $55^{\circ} \mathrm{C}$ and $30 \mathrm{~s}$ at $72^{\circ} \mathrm{C}$. Water was used instead of template 
DNA for a negative control. The PCR products were then evaluated by $2 \%$ agarose gel electrophoresis, after which they were extracted from the gels and purified using a HiYieldTMGel/PCR DNA Extraction Kit (RBC bioscience, Taipei, Taiwan). The purified PCR products were processed for the DNA sequencing reaction using the ABI-PRISM BigDye Terminator version 3.1 (Applied Biosystems, Foster, CA, USA) according to manufacturers' protocol. Sequencing was performed in both directions using the same primers used for PCR. Sequencing reactions were performed with 25 cycles for $10 \mathrm{~s}$ at $96^{\circ} \mathrm{C}$, $5 \mathrm{~s}$ at $50^{\circ} \mathrm{C}$, and $4 \mathrm{~min}$ at $60^{\circ} \mathrm{C}$. Sequence data were generated using the ABI PRISM 3100 DNA Analyzer (Applied Biosystems) and the sequences were then analyzed and compared using the Sequencer 3.1.1. software (Applied Biosystems).

\section{Statistical Analysis}

A $\chi^{2}$ or Fisher's exact test was used to analyze the relationships of lymph node metastasis status with each predictive factor. Factors independently associated with a risk of nodal metastasis were tested by means of stepwise logistic regression analysis. A $P$-value of less than 0.05 was considered to be statistically significant. All the calculations were performed using the statistical software package SPSS (version 13.0 SPSS Inc., Chicago, IL, USA).

\section{Results}

Of 210 patients with papillary thyroid carcinomas, $147(70 \%)$ had small papillary carcinomas $\leq 10 \mathrm{~mm}$ (papillary thyroid microcarcinoma). Of the 210 papillary thyroid carcinoma patients, the proportions of lymph node metastases were 54 and $6 \%$ in the central and lateral compartments, respectively. There was no skip metastasis, which was defined as lateral lymph node metastasis in the absence of central lymph node metastasis. Of the 147 papillary thyroid microcarcinoma patients, the rate of lymph node metastases was 44 and $3 \%$ in the central and lateral compartments, respectively.

Cyclin D1 was constantly expressed as an invasive tumor front, although the percentage of cyclin D1-positive tumor cells varied greatly in the whole tissue sections of the 210 papillary thyroid carcinoma cases (grade $1,1.4 \%$; grade $2,11.4 \%$; grade 3 , $11.0 \%$; grade $4,9.5 \%$; grade $5,66.7 \%$ ). Normalappearing follicular epithelial cells were negative for cyclin D1. Hürthle cells were occasionally positive for cyclin D1 in lymphocytic thyroiditis; however, the staining intensity was weaker than in papillary thyroid carcinoma cells and the distribution of staining was not diffuse, but focal. The tumor cells showing strong nuclear expression of cyclin D1 were also positive in the cytoplasm, whereas the cytoplasmic expression was never observed in nonneoplastic cells (Figure 1). Therefore, we could define the exact extent of tumor spread using the
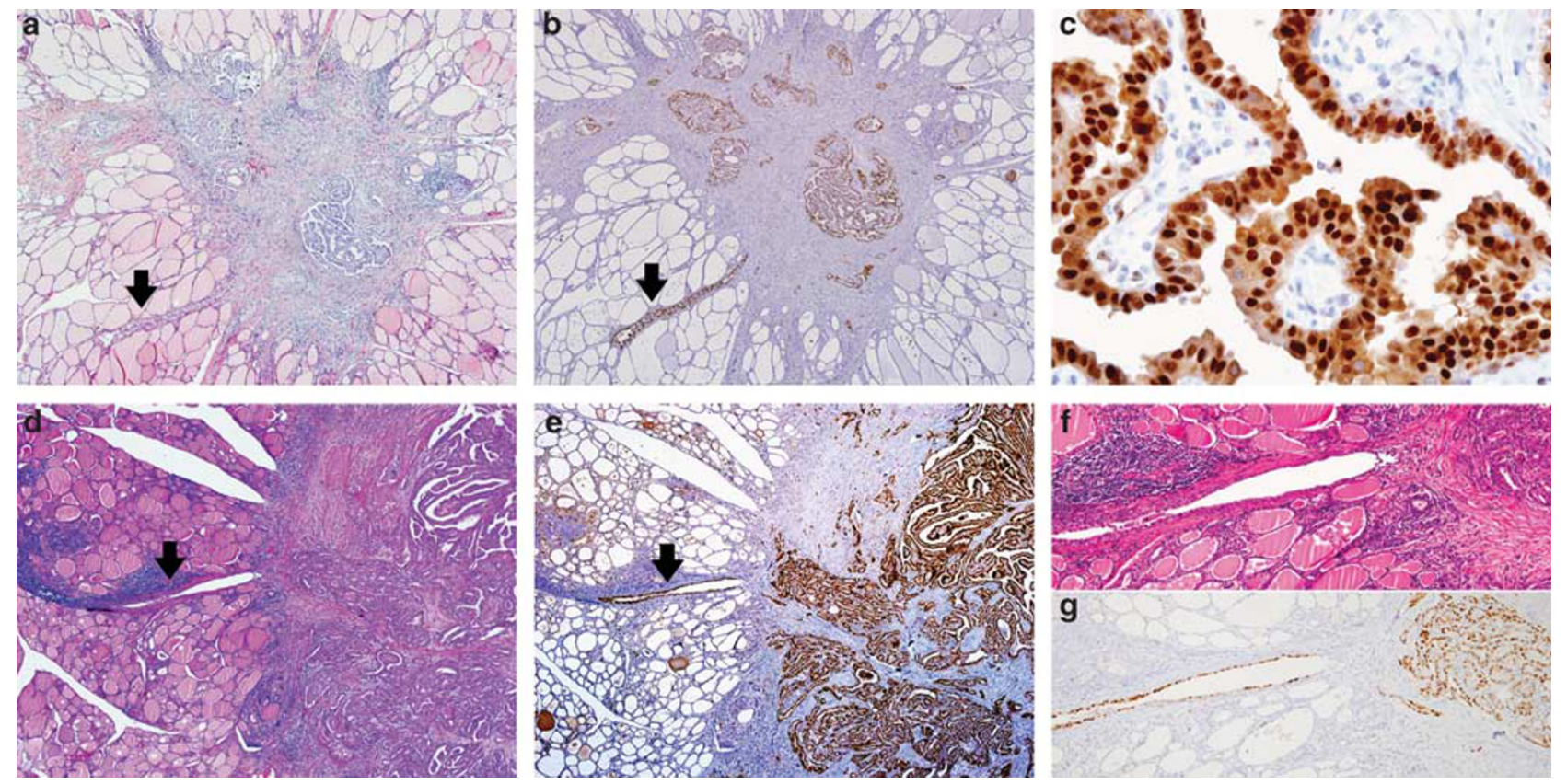

Figure 1 (a) A 0.4-cm-sized papillary microcarcinoma. (b) Cyclin D1 expression in the same tissue section shown in (a). Most of the tumor cells are strongly positive for cyclin D1, whereas surrounding normal thyroid tissue is negative. (c) The tumor cells show nuclear and cytoplasmic immunoreactivity for cyclin D1. Arrows indicate a pattern of laterally spreading tubular growth along the fibrous septa around the invasive front of the tumor in (a), (b), (d) and (e). (d) A 0.6-cm-sized papillary microcarcinoma with Hashimoto thyroiditis. It is difficult to identify the lateral tubular growth morphologically (d), but the growth pattern can be easily detected by cyclin D1 staining $(\mathbf{e}, \mathbf{g})$. (f, g) Magnified views of the lateral tubular growth in the same case shown in (d, e). 

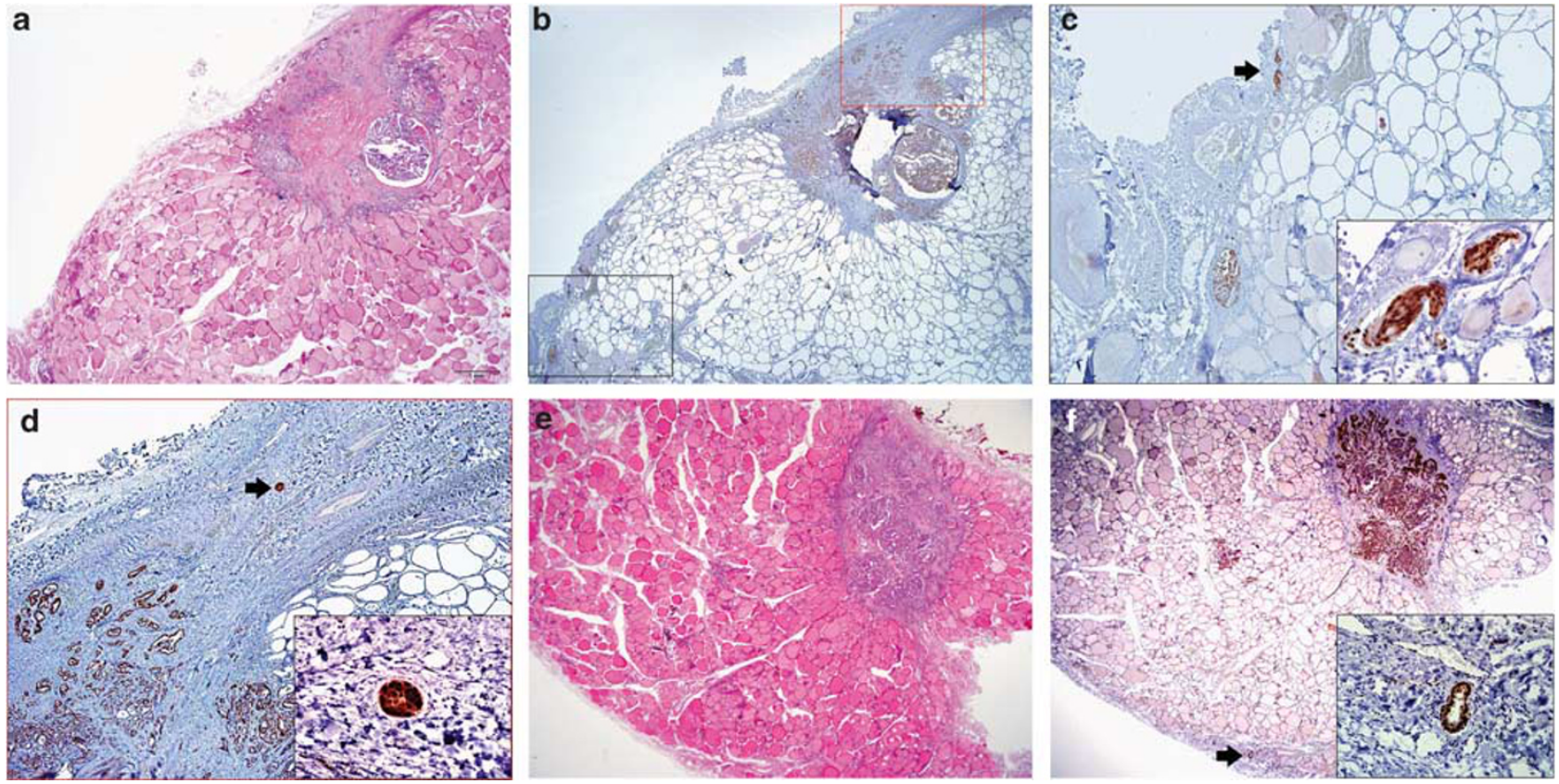

Figure 2 (a) A 0.6-cm-sized papillary microcarcinoma. (b) Cyclin D1 expression in the same tissue section shown in (a). Most of the tumor cells are strongly positive for cyclin D1, whereas surrounding normal thyroid tissue is negative. (c, d) Magnified views corresponding to the two inboxes (c, black color line; d, red color line) in (b). Cautery artifact produces distorted morphology, rendering accurate diagnosis of invasion difficult at the surgical resection margin. (c) Cyclin D1 immunostain highlights the tumor cells (arrow) in areas exhibiting cautery artifact well. (d) There is an isolated gland (arrow) of tumor cells that is more than $500 \mu \mathrm{m}$ from the invasive front. (e) A 0.5-cm-sized papillary microcarcinoma. (f) Cyclin D1 immunostain in the same section shown in (e). One isolated gland of tumor cells (arrow) is noted.

cyclin D1 staining to detect the outer margins of the tumor. The consistent immunostaining for cyclin D1 at the invasive front revealed a laterally spreading tubular growth along the fibrous septa around the invasive front of the tumor (lateral tubular growth, Figure 1) and individual isolated glands of tumor cells in the extra-tumoral region (isolated glands, Figure 2). We defined the isolated glands as individual glands of papillary thyroid carcinoma cells found in areas a minimum of $500 \mu \mathrm{m}$ apart from the primary tumor. Although the growth patterns of lateral tubular growth and isolated glands were easily identified in cyclin D1 immunostained slides, they were very difficult to detect in routine hematoxylin-eosin-stained slides.

The $B R A F^{\mathrm{V} 600 \mathrm{E}}$ mutation was observed in 162 of $210(77 \%)$ patients with papillary thyroid carcinoma (Table 1).

\section{Univariate Analysis}

Papillary thyroid carcinomas with lymph node metastases were characterized by an age of less than 45 years $(P<0.001)$, large tumor size $(>10 \mathrm{~mm}$, $P<0.001)$, non-follicular variant $(P=0.005)$, invasive growth pattern $(P=0.007)$, isolated glands $(P<0.001)$, lateral tubular growth $(P<0.001)$, extrathyroid extension $(P=0.006)$ and multifocality $(P=0.005)$ (Table 1). In addition, we analyzed the relationship between the clinicopathological parameters and lymph node metastasis in 147 papillary thyroid microcarcinomas. The patients were divided into two groups with a cutoff point of $0.5 \mathrm{~cm}$ according to the tumor size of the papillary thyroid microcarcinoma. A young age $(<45$ years, $P<0.001)$, invasive growth pattern $(P=0.001)$, isolated glands $(P<0.001)$, lateral tubular growth $(P<0.001)$ and multifocality $(P=0.005)$ were more frequently observed in papillary thyroid microcarcinoma with lymph node metastasis than those without lymph node metastasis (Table 2). There was no significant relationship between $B R A F^{\mathrm{v} 600 \mathrm{E}}$ mutation and lymph node metastasis in papillary thyroid carcinoma or papillary thyroid microcarcinoma.

The presence of a $B R A F^{\mathrm{V} 600 \mathrm{E}}$ mutation was significantly associated with extrathyroid extension $(P=0.007)$ and a lower frequency of concurrent Hashimoto thyroiditis $(P=0.023)$; however, other clinicopathological parameters were not related to the mutation (Table 3).

\section{Multivariate Analysis}

To identify independent predictors of lymph node metastases in papillary thyroid carcinoma, multivariate analysis using stepwise logistic regression was conducted on parameters shown to be significant upon univariate analysis. Of the 210 papillary thyroid carcinomas, young age $(<45$ years, 
Table 1 Predictive factors of lymph node metastasis in 210 papillary thyroid carcinomas

\begin{tabular}{|c|c|c|c|c|}
\hline & \multicolumn{2}{|c|}{ Lymph node metastasis } & \multirow[b]{2}{*}{$\mathrm{P}$-value } & \multirow{2}{*}{$\begin{array}{c}\text { Total } \\
(\mathrm{n}=210)\end{array}$} \\
\hline & No $(\mathrm{n}=97)$ & Yes $(\mathrm{n}=113)$ & & \\
\hline \multicolumn{5}{|l|}{ Age } \\
\hline$<45$ years & $24(25 \%)$ & $58(51 \%)$ & \multirow[t]{2}{*}{$<0.001$} & $82(39 \%)$ \\
\hline$\geq 45$ years & $73(75 \%)$ & $55(49 \%)$ & & $128(61 \%)$ \\
\hline \multicolumn{5}{|l|}{ Gender } \\
\hline Female & $84(87 \%)$ & $90(80 \%)$ & \multirow[t]{2}{*}{0.183} & $174(83 \%)$ \\
\hline Male & $13(13 \%)$ & $23(20 \%)$ & & $36(17 \%)$ \\
\hline \multicolumn{5}{|l|}{ Side } \\
\hline Right lobe & $48(50 \%)$ & $43(38 \%)$ & \multirow[t]{3}{*}{0.134} & $91(43 \%)$ \\
\hline Left lobe & $35(36 \%)$ & $43(38 \%)$ & & $78(37 \%)$ \\
\hline Both lobes & $14(14 \%)$ & $27(24 \%)$ & & $41(20 \%)$ \\
\hline \multicolumn{5}{|l|}{ Tumor size } \\
\hline$\leq 10 \mathrm{~mm}$ & $83(86 \%)$ & $64(57 \%)$ & \multirow[t]{2}{*}{$<0.001$} & $147(70 \%)$ \\
\hline$>10 \mathrm{~mm}$ & $14(14 \%)$ & $49(43 \%)$ & & $63(30 \%)$ \\
\hline \multicolumn{5}{|l|}{ Follicular variant } \\
\hline No & $83(86 \%)$ & 109 (96\%) & \multirow[t]{2}{*}{0.005} & $192(91 \%)$ \\
\hline Yes & $14(14 \%)$ & $4(4 \%)$ & & $18(9 \%)$ \\
\hline \multicolumn{5}{|l|}{ Growth pattern } \\
\hline Invasive & $72(74 \%)$ & $100(89 \%)$ & \multirow[t]{2}{*}{0.007} & $172(82 \%)$ \\
\hline Expansive & $25(26 \%)$ & $13(11 \%)$ & & $38(18 \%)$ \\
\hline \multicolumn{5}{|l|}{ Isolated glands ${ }^{\mathrm{a}}$} \\
\hline No & $75(77 \%)$ & $27(24 \%)$ & \multirow[t]{2}{*}{$<0.001$} & $102(49 \%)$ \\
\hline Yes & $22(23 \%)$ & $86(76 \%)$ & & $108(51 \%)$ \\
\hline \multicolumn{5}{|c|}{ Lateral tubular growth ${ }^{\mathrm{a}}$} \\
\hline No & $84(87 \%)$ & $43(38 \%)$ & \multirow{2}{*}{$<0.001$} & $127(60 \%)$ \\
\hline Yes & $13(13 \%)$ & $70(62 \%)$ & & $83(40 \%)$ \\
\hline \multicolumn{5}{|c|}{ Extrathyroid extension } \\
\hline No & $48(50 \%)$ & $35(31 \%)$ & \multirow[t]{2}{*}{0.006} & $83(40 \%)$ \\
\hline Yes & $49(50 \%)$ & $78(69 \%)$ & & $127(60 \%)$ \\
\hline \multicolumn{5}{|l|}{ Multifocality } \\
\hline No & $73(75 \%)$ & $64(57 \%)$ & \multirow[t]{2}{*}{0.005} & $137(65 \%)$ \\
\hline Yes & $24(25 \%)$ & $49(43 \%)$ & & $73(35 \%)$ \\
\hline \multicolumn{5}{|l|}{ Cyclin D1 expression } \\
\hline Low (0-25\%) & $17(18 \%)$ & $10(9 \%)$ & \multirow[t]{2}{*}{0.061} & $27(13 \%)$ \\
\hline High (26-100\%) & $80(83 \%)$ & $103(91 \%)$ & & $183(87 \%)$ \\
\hline \multicolumn{5}{|l|}{ BRAF ${ }^{V 600 E}$ mutation } \\
\hline No & $22(23 \%)$ & $26(23 \%)$ & \multirow[t]{2}{*}{0.955} & $48(23 \%)$ \\
\hline Yes & $75(77 \%)$ & $87(77 \%)$ & & $162(77 \%)$ \\
\hline
\end{tabular}

${ }^{\mathrm{a}}$ Tumor growth patterns identified by cyclin D1 staining.

$P=0.001, \mathrm{OR}=5.146)$, larger tumor size $(>10 \mathrm{~mm}$, $P=0.034, \mathrm{OR}=3.119)$, isolated glands $(P<0.001$, $\mathrm{OR}=21.042)$ and lateral tubular growth $(P<0.001$, $\mathrm{OR}=24.652$ ) were independent predictors of lymph node metastasis (Table 4). In the 147 papillary thyroid microcarcinomas, young age $(<45$ years, $P=0.001, \mathrm{OR}=6.353)$, isolated glands $(P<0.001$, $\mathrm{OR}=17.937)$ and lateral tubular growth $(P<0.001$, $\mathrm{OR}=25.729$ ) were independent predictors of lymph node metastasis (Table 5).
Table 2 Predictive factors of lymph node metastasis in 147 papillary thyroid microcarcinomas

\begin{tabular}{|c|c|c|c|c|}
\hline & \multicolumn{2}{|c|}{ Lymph node metastasis } & \multirow[b]{2}{*}{$\mathrm{P}$-value } & \multirow{2}{*}{$\begin{array}{c}\text { Total } \\
(\mathrm{n}=147)\end{array}$} \\
\hline & No $(\mathrm{n}=83)$ & Yes $(\mathrm{n}=64)$ & & \\
\hline \multicolumn{5}{|l|}{ Age } \\
\hline$<45$ years & $21(25 \%)$ & $36(56 \%)$ & \multirow[t]{2}{*}{$<0.001$} & $57(39 \%)$ \\
\hline$\geq 45$ years & $26(75 \%)$ & $28(44 \%)$ & & $90(61 \%)$ \\
\hline \multicolumn{5}{|l|}{ Gender } \\
\hline Female & $72(87 \%)$ & $54(84 \%)$ & \multirow[t]{2}{*}{0.684} & $126(86 \%)$ \\
\hline Male & $11(13 \%)$ & $10(16 \%)$ & & $21(14 \%)$ \\
\hline \multicolumn{5}{|l|}{ Side } \\
\hline Right lobe & $42(51 \%)$ & $27(42 \%)$ & \multirow[t]{3}{*}{0.598} & $69(47 \%)$ \\
\hline Left lobe & $30(36 \%)$ & $27(42 \%)$ & & $57(39 \%)$ \\
\hline Both lobes & $11(13 \%)$ & $10(16 \%)$ & & $21(14 \%)$ \\
\hline \multicolumn{5}{|l|}{ Tumor size } \\
\hline$<5 \mathrm{~mm}$ & $12(15 \%)$ & $10(16 \%)$ & \multirow[t]{2}{*}{0.844} & $22(15 \%)$ \\
\hline $5-10 \mathrm{~mm}$ & $71(85 \%)$ & $54(84 \%)$ & & $125(85 \%)$ \\
\hline \multicolumn{5}{|l|}{ Growth pattern } \\
\hline Invasive & 59 (71\%) & $59(92 \%)$ & \multirow[t]{2}{*}{0.001} & $118(80 \%)$ \\
\hline Expansive & $24(29 \%)$ & $5(8 \%)$ & & $29(20 \%)$ \\
\hline \multicolumn{5}{|l|}{ Follicular variant } \\
\hline No & $72(87 \%)$ & $61(95 \%)$ & \multirow[t]{2}{*}{0.079} & $133(90 \%)$ \\
\hline Yes & $11(13 \%)$ & $3(5 \%)$ & & $14(10 \%)$ \\
\hline \multicolumn{5}{|l|}{ Isolated glands ${ }^{\mathrm{a}}$} \\
\hline No & $65(78 \%)$ & $24(38 \%)$ & \multirow[t]{2}{*}{$<0.001$} & $89(60 \%)$ \\
\hline Yes & $18(22 \%)$ & $40(62 \%)$ & & $58(40 \%)$ \\
\hline \multicolumn{5}{|c|}{ Lateral tubular growth ${ }^{\mathrm{a}}$} \\
\hline No & $71(85 \%)$ & $23(36 \%)$ & \multirow{2}{*}{$<0.001$} & $94(64 \%)$ \\
\hline Yes & $12(15 \%)$ & $41(64 \%)$ & & $53(36 \%)$ \\
\hline \multicolumn{5}{|c|}{ Extrathyroid extension } \\
\hline No & $44(53 \%)$ & $25(39 \%)$ & \multirow[t]{2}{*}{0.093} & $69(47 \%)$ \\
\hline Yes & $39(47 \%)$ & $39(61 \%)$ & & $78(53 \%)$ \\
\hline \multicolumn{5}{|l|}{ Multifocality } \\
\hline No & $64(77 \%)$ & $39(61 \%)$ & \multirow[t]{2}{*}{0.034} & $103(70 \%)$ \\
\hline Yes & $19(23 \%)$ & $25(39 \%)$ & & $44(30 \%)$ \\
\hline \multicolumn{5}{|l|}{ Cyclin D1 expression } \\
\hline Low $(0-25 \%)$ & $15(18 \%)$ & $6(9 \%)$ & \multirow[t]{2}{*}{0.135} & $21(14 \%)$ \\
\hline High $(26-100 \%)$ & $68(82 \%)$ & $58(91 \%)$ & & $126(86 \%)$ \\
\hline \multicolumn{5}{|l|}{ BRAF $F^{V 600 E}$ mutation } \\
\hline No & $18(22 \%)$ & $19(30 \%)$ & \multirow[t]{2}{*}{0.268} & $37(25 \%)$ \\
\hline Yes & $65(78 \%)$ & $45(70 \%)$ & & $110(75 \%)$ \\
\hline
\end{tabular}

${ }^{\mathrm{a}}$ Tumor growth patterns identified by cyclin D1 staining.

\section{Discussion}

We demonstrated that the rate of lymph node metastasis was independently related to the age, tumor size and unique tumor growth patterns verified by cyclin D1 staining in a series of 210 papillary thyroid carcinomas, including 147 papillary thyroid microcarcinomas.

Upon multivariate analysis of the clinicopathological risk factors, lymph node metastasis was more common among patients younger than age 45 years. 
Table 3 Correlation between $B R A F^{\mathrm{V} 600 \mathrm{E}}$ mutation and clinicopathological values in 210 Korean patients with papillary thyroid carcinoma

\begin{tabular}{|c|c|c|c|c|}
\hline & \multicolumn{2}{|c|}{$B R A F^{V G 00 E}$ mutation } & \multirow[b]{2}{*}{$\mathrm{P}$-value } & \multirow{2}{*}{$\begin{array}{c}\text { Total } \\
(\mathrm{n}=210)\end{array}$} \\
\hline & No $(\mathrm{n}=48)$ & Yes $(\mathrm{n}=162)$ & & \\
\hline \multicolumn{5}{|l|}{ Age } \\
\hline$<45$ years & $16(33 \%)$ & $66(41 \%)$ & 0.356 & $82(39 \%)$ \\
\hline$\geq 45$ years & $32(67 \%)$ & $96(59 \%)$ & & $128(61 \%)$ \\
\hline \multicolumn{5}{|l|}{ Gender } \\
\hline Female & $41(85 \%)$ & $133(82 \%)$ & 0.592 & $174(83 \%)$ \\
\hline Male & $7(15 \%)$ & $29(18 \%)$ & & $36(17 \%)$ \\
\hline \multicolumn{5}{|l|}{ Side } \\
\hline Right lobe & $22(46 \%)$ & $69(43 \%)$ & 0.924 & $91(43 \%)$ \\
\hline Left lobe & $17(35 \%)$ & $61(38 \%)$ & & $78(37 \%)$ \\
\hline Both lobes & $9(19 \%)$ & $32(20 \%)$ & & $41(20 \%)$ \\
\hline \multicolumn{5}{|l|}{ Tumor size } \\
\hline$<5 \mathrm{~mm}$ & $37(77 \%)$ & $110(68 \%)$ & 0.223 & $147(70 \%)$ \\
\hline $5-10 \mathrm{~mm}$ & $11(23 \%)$ & $52(32 \%)$ & & $63(30 \%)$ \\
\hline \multicolumn{5}{|l|}{ Growth pattern } \\
\hline Invasive & $40(83 \%)$ & $132(82 \%)$ & 0.770 & $172(82 \%)$ \\
\hline Expansive & $8(17 \%)$ & $30(19 \%)$ & & $38(18 \%)$ \\
\hline \multicolumn{5}{|l|}{ Follicular variant } \\
\hline No & $44(92 \%)$ & $148(91 \%)$ & 1.000 & $192(91 \%)$ \\
\hline Yes & $4(8 \%)$ & $14(9 \%)$ & & $18(9 \%)$ \\
\hline \multicolumn{5}{|l|}{ Isolated glands ${ }^{\mathrm{a}}$} \\
\hline No & $23(48 \%)$ & $79(49 \%)$ & 0.918 & $102(49 \%)$ \\
\hline Yes & $25(52 \%)$ & $83(51 \%)$ & & $108(51 \%)$ \\
\hline \multicolumn{5}{|c|}{ Lateral tubular growth $^{\mathrm{a}}$} \\
\hline No & $34(71 \%)$ & $93(58 \%)$ & 0.095 & $127(60 \%)$ \\
\hline Yes & $14(30 \%)$ & $69(43 \%)$ & & $83(40 \%)$ \\
\hline \multicolumn{5}{|c|}{ Extrathyroid extension } \\
\hline No & $27(56 \%)$ & $56(35 \%)$ & 0.007 & $83(40 \%)$ \\
\hline Yes & $21(44 \%)$ & $106(65 \%)$ & & $127(60 \%)$ \\
\hline \multicolumn{5}{|l|}{ Multifocality } \\
\hline No & $36(75 \%)$ & $101(62 \%)$ & 0.106 & $137(65 \%)$ \\
\hline Yes & $12(25 \%)$ & $61(38 \%)$ & & $73(35 \%)$ \\
\hline \multicolumn{5}{|c|}{ Hashimoto thyroiditis } \\
\hline No & $40(83 \%)$ & $152(94 \%)$ & 0.023 & $192(91 \%)$ \\
\hline Yes & $8(17 \%)$ & $10(6 \%)$ & & $18(9 \%)$ \\
\hline \multicolumn{5}{|l|}{ Cyclin D1 expression } \\
\hline Low $(0-25 \%)$ & $6(13 \%)$ & $21(13 \%)$ & 0.933 & $27(13 \%)$ \\
\hline High (26-100\%) & $42(88 \%)$ & $141(87 \%)$ & & $183(87 \%)$ \\
\hline \multicolumn{5}{|c|}{ Lymph node metastasis } \\
\hline No & $22(46 \%)$ & $75(46 \%)$ & 0.955 & $97(46 \%)$ \\
\hline Yes & $26(54 \%)$ & $87(54 \%)$ & & $113(54 \%)$ \\
\hline
\end{tabular}

${ }^{\mathrm{a}}$ Tumor growth patterns identified by cyclin D1 staining.

This finding is consistent with the results of a previous study conducted by Machens et al. ${ }^{17}$

Papillary thyroid carcinomas frequently present with multifocal tumors in up to $80 \%$ of patients; ${ }^{18}$ however, there is currently debate about whether tumor multifocality in papillary thyroid carcinoma represents intraglandular spread of a single tumor or
Table 4 Multivariate logistic regression analysis of factors predictive of lymph node metastasis in the 210 papillary thyroid carcinomas evaluated in this study

\begin{tabular}{lrrc}
\hline Variables & P-value & $\begin{array}{c}\text { Odds } \\
\text { ratio }\end{array}$ & $\begin{array}{c}\text { 95\% Confidence } \\
\text { interval }\end{array}$ \\
\hline Age $(<45$ years) & 0.001 & 5.146 & $2.001-13.233$ \\
Tumor size ( $>10 \mathrm{~mm})$ & 0.034 & 3.119 & $1.088-8.941$ \\
Non-follicular variant & 0.284 & 2.516 & $0.465-13.618$ \\
Invasive growth pattern $^{\text {Isolated glands }}$ & 0.966 & 1.025 & $0.323-3.254$ \\
Lateral tubular growth $^{\mathrm{a}}$ & $<0.001$ & 21.042 & $7.418-59.690$ \\
Extrathyroid extension & 0.001 & 24.652 & $8.080-75.210$ \\
Multifocality & 0.349 & 1.319 & $0.533-3.266$ \\
& & 1.614 & $0.651-4.002$ \\
\hline
\end{tabular}

${ }^{\mathrm{a}}$ Tumor growth patterns identified by cyclin D1 staining.

Table 5 Multivariate logistic regression analysis of predictive factors of the lymph node metastasis in the 147 papillary thyroid microcarcinomas evaluated in this study

\begin{tabular}{lrrc}
\hline Variables & P-value & $\begin{array}{c}\text { Odds } \\
\text { ratio }\end{array}$ & $\begin{array}{c}\text { 95\% Confidence } \\
\text { interval }\end{array}$ \\
\hline Age (<45 years) & 0.001 & 6.353 & $2.177-18.540$ \\
Tumor size (5-10 mm) & 0.155 & 0.370 & $0.094-1.457$ \\
Non-follicular variant & 0.520 & 1.824 & $0.292-11.383$ \\
Invasive growth pattern & 0.468 & 1.701 & $0.405-7.139$ \\
Isolated glands $^{\mathrm{a}}$ & $<0.001$ & 17.937 & $5.162-62.324$ \\
Lateral tubular growth $^{\mathrm{a}}$ & $<0.001$ & 25.729 & $7.343-90.143$ \\
Extrathyroid extension & 0.459 & 1.488 & $0.520-4.261$ \\
Multifocality & 0.215 & 2.030 & $0.663-6.212$
\end{tabular}

${ }^{\mathrm{a}}$ Tumor growth patterns identified by cyclin D1 staining.

de novo occurrence of distinct tumors. In this study of 210 papillary thyroid carcinomas, tumor multifocality was found to be more common with increasing tumor size $(<5 \mathrm{~mm}, 9 \%$; 5-10 mm, $34 \%$; $>10 \mathrm{~mm}, 46 \% ; P=0.007)$ and associated with lymph node metastasis upon univariate analysis $(P=0.007)$, but not by multivariate analysis. These results suggest that multifocality may occur during the progression or spread of papillary thyroid carcinoma and represent intraglandular dissemination of the primary tumor, at least in some cases. A recent study showed that among papillary thyroid microcarcinomas with multiple tumor foci, $83 \%$ had genetic alterations consistent with monoclonal origin based on genome-wide allelotyping and $B R A F$ mutation analysis. ${ }^{18}$ The authors also suggested that papillary thyroid microcarcinomas were most often monoclonally derived and that multiple foci developed through the intraglandular spread of an original tumor.

All of the tumors chosen for this study were cyclin D1 positive and these results are consistent with a previous study conducted by Saiz et al. ${ }^{19}$ Overexpression of cyclin D1 has been identified as a predictor of lymph node metastasis in papillary thyroid carcinoma; ${ }^{7,8,10}$ however, we found no significant correlation between its overexpression 
(more than $25 \%$ of positively staining tumor cells) and lymph node metastasis $(P=0.061)$.

The isolated glands, which are defined as individual glands of papillary thyroid carcinoma cells found in the extra-tumoral region, are distinctive histological features of papillary thyroid carcinoma and should not be confused with the tumor multifocal foci. Isolated glands are composed of a lumen lined by a row of papillary carcinoma cells and can be detected by cyclin D1 immunohistochemical staining. The lateral tubular growth is defined as a laterally spreading tubular growth along the fibrous septa around the invasive front of the tumor. Although invasive-type papillary thyroid carcinomas showed more frequent lateral tubular growth than expansive-type papillary thyroid carcinomas $(P=0.010)$, lateral tubular growth does not indicate an invasive growth pattern. Indeed, lateral tubular growth can be found in thick encapsulated papillary thyroid carcinomas. Thyroid gland has a rich network of intraglandular lymphatics that intercommunicate between lobes through the isthmus and drain to the cervical lymph nodes. ${ }^{4}$ Intraglandular metastasis and cervical lymph node metastasis occur through these rich lymphatic networks. Multivariate analysis showed isolated glands and lateral tubular growth to be independent risk factors for lymph node metastasis. To determine if isolated glands and lateral tubular growth represented the lymphatic invasion of tumor cells, we used D2-40 immunostaining to identify lymphatic vessels. D2-40 staining revealed that isolated glands were not found in lymphatic spaces, but lateral tubular growth was found within lymphatic vessels in only some cases (data not shown).

Our predictive system may be a useful predictive tool in clinical practice. For example, when thyroid lobectomy alone was conducted for the treatment of small, low-risk, isolated, intrathyroidal papillary carcinomas, our predictive system may predict the risk of cervical nodal metastasis, and the lymph node status will then influence patient treatment decision-making.

We found that the $B R A F^{\mathrm{V} 600 \mathrm{E}}$ mutation was detected in $77 \%$ of Korean patients with papillary thyroid carcinoma. However, the mutation was not significantly associated with age at diagnosis, gender, tumor size, tumor growth pattern, follicular variant, multifocality or lymph node metastasis. These findings are consistent with those of a previous study in which the mutation was not associated with poor prognostic factors in Korean patients with papillary thyroid carcinoma. ${ }^{15}$ In Korea, $B R A F^{\mathrm{V} 600 \mathrm{E}}$ mutation has been detected at a greater frequency in papillary thyroid carcinoma without concurrent Hashimoto thyroiditis than in papillary thyroid carcinoma with Hashimoto thyroiditis. ${ }^{20,21}$ In this study, the mutation was found in $10(56 \%)$ of 18 patients with background Hashimoto thyroiditis, but in 152 (79\%) of 192 patients without Hashimoto thyroiditis (Table 3). To establish the correlation between Hashimoto thyroiditis, papillary thyroid carcinoma and $B R A F^{\mathrm{V} 600 \mathrm{E}}$ mutation, it is necessary to study as many cases of coexistent Hashimoto thyroiditis with papillary thyroid carcinoma as possible.

In summary, we found that cyclin D1 staining could define the exact extent of tumor spread that was used for identifying isolated glands and lateral tubular growth. These growth patterns were novel independent predictors of lymph node metastasis in papillary thyroid carcinoma. The results produced here may be useful during progress assessment and contribute to the design of an appropriate treatment for individual patients.

\section{Acknowledgement}

This study was supported by a grant from the Catholic Comprehensive Cancer Institute of the Catholic University of Korea in 2009.

\section{Disclosure/conflict of interest}

The authors declare no conflict of interest.

\section{References}

1 Ministry of Health and Welfare. 2005 Annual report of the Korea Central Cancer Registry. 2008 October, 2008 [cited 2009 October 30]; available from:http://ncc.re. kr/manage/manage03_033_view.jsp?bbsnum $=111$.

2 Enewold L, Zhu K, Ron E, et al. Rising thyroid cancer incidence in the United States by demographic and tumor characteristics, 1980-2005. Cancer Epidemiol Biomarkers Prev 2009;18:784-791.

3 Kim SK, Kim DL, Han HS, et al. Pyrosequencing analysis for detection of a BRAFV600E mutation in an FNAB specimen of thyroid nodules. Diagn Mol Pathol 2008;17:118-125.

4 Katoh R, Sasaki J, Kurihara H, et al. Multiple thyroid involvement (intraglandular metastasis) in papillary thyroid carcinoma. A clinicopathologic study of 105 consecutive patients. Cancer 1992;70:1585-1590.

5 Dionigi G, Dionigi R, Bartalena L, et al. Surgery of lymph nodes in papillary thyroid cancer. Expert Rev Anticancer Ther 2006;6:1217-1229.

6 Leboulleux S, Rubino C, Baudin E, et al. Prognostic factors for persistent or recurrent disease of papillary thyroid carcinoma with neck lymph node metastases and/or tumor extension beyond the thyroid capsule at initial diagnosis. J Clin Endocrinol Metab 2005;90: 5723-5729.

7 Khoo ML, Ezzat S, Freeman JL, et al. Cyclin D1 protein expression predicts metastatic behavior in thyroid papillary microcarcinomas but is not associated with gene amplification. J Clin Endocrinol Metab 2002; 87:1810-1813.

8 Khoo ML, Beasley NJ, Ezzat S, et al. Overexpression of cyclin D1 and underexpression of p27 predict lymph node metastases in papillary thyroid carcinoma. J Clin Endocrinol Metab 2002;87:1814-1818. 
9 Ito Y, Uruno T, Takamura Y, et al. Papillary microcarcinomas of the thyroid with preoperatively detectable lymph node metastasis show significantly higher aggressive characteristics on immunohistochemical examination. Oncology 2005;68:87-96.

10 Pesutic-Pisac V, Punda A, Gluncic I, et al. Cyclin D1 and p27 expression as prognostic factor in papillary carcinoma of thyroid: association with clinicopathological parameters. Croat Med J 2008; 49:643-649.

11 Min HS, Choe G, Kim SW, et al. S100A4 expression is associated with lymph node metastasis in papillary microcarcinoma of the thyroid. Mod Pathol 2008;21:748-755.

12 Xing M. BRAF mutation in thyroid cancer. Endocr Relat Cancer 2005;12:245-262.

13 Xing M. BRAF mutation in papillary thyroid cancer: pathogenic role, molecular bases, and clinical implications. Endocr Rev 2007;28:742-762.

14 Jung CK, Choi YJ, Lee KY, et al. The cytological, clinical, and pathological features of the cribriformmorular variant of papillary thyroid carcinoma and mutation analysis of CTNNB1 and BRAF genes. Thyroid 2009;19:905-913.

15 Kim TY, Kim WB, Song JY, et al. The BRAF mutation is not associated with poor prognostic factors in Korean patients with conventional papillary thyroid microcarcinoma. Clin Endocrinol (Oxf) 2005;63:588-593.

16 Lee JH, Lee ES, Kim YS, et al. BRAF mutation and AKAP9 expression in sporadic papillary thyroid carcinomas. Pathology 2006;38:201-204.

17 Machens A, Holzhausen HJ, Lautenschlager C, et al. Enhancement of lymph node metastasis and distant metastasis of thyroid carcinoma. Cancer 2003;98: $712-719$

18 Jovanovic L, Delahunt B, McIver B, et al. Most multifocal papillary thyroid carcinomas acquire genetic and morphotype diversity through subclonal evolution following the intra-glandular spread of the initial neoplastic clone. J Pathol 2008;215:145-154.

19 Saiz AD, Olvera M, Rezk S, et al. Immunohistochemical expression of cyclin D1, E2F-1, and Ki-67 in benign and malignant thyroid lesions. J Pathol 2002; 198:157-162.

$20 \mathrm{Kim}$ SK, Song KH, Lim SD, et al. Clinical and pathological features and the BRAF(V600E) mutation in patients with papillary thyroid carcinoma with and without concurrent Hashimoto thyroiditis. Thyroid 2009;19:137-141.

$21 \mathrm{Kim} \mathrm{KH}$, Suh KS, Kang DW, et al. Mutations of the BRAF gene in papillary thyroid carcinoma and in Hashimoto's thyroiditis. Pathol Int 2005;55:540-545. 